\title{
Certain new proportional and Hadamard proportional fractional integral inequalities
}

Gauhar Rahman ${ }^{1}$, Kottakkaran Sooppy Nisar ${ }^{2}$ and Thabet Abdeljawad ${ }^{3,4,5^{*}}$ (i)

\section{"Correspondence:}

tabdeljawad@psu.edu.sa

${ }^{3}$ Department of Mathematics and

General Sciences, Prince Sultan

University, Riyadh, Kingdom of

Saudi Arabia

${ }^{4}$ Department of Medical Research, China Medical University, Taichung 40402, Taiwan

Full list of author information is available at the end of the article

\begin{abstract}
The main goal of this paper is estimating certain new fractional integral inequalities for the extended Chebyshev functional in the sense of synchronous functions by employing proportional fractional integral (PFI) and Hadamard proportional fractional integral. We establish certain inequalities concerning one- and two-parameter proportional and Hadamard proportional fractional integrals. We also discuss certain particular cases.
\end{abstract}

MSC: 26A33;26D10;26D53

Keywords: Fractional integrals; Proportional fractional integrals; Hadamard proportional fractional integrals; Fractional integral inequalities; Chebyshev functional

\section{Introduction}

The integral inequalities play a major role in the field of differential equations and applied mathematics. Applications of integral inequalities are found in applied sciences, such as statistical problems, transform theory, numerical quadrature, and probability. In the last few years, many researchers have established various types of integral inequalities by employing different approaches. The interested readers are suggested to see $[4,5,8,14,15,17,18]$. In $[28,39-41]$ the researchers established different kinds of integral inequalities by employing various types of fractional integrals.

In the last few years, the field of fractional calculus has been extensively studied due to wide applications in diverse domains. Several different kinds of fractional integral and derivative operators have been investigated. We refer the readers to $[1-3,6,7,11,21,24]$. In $[20,22]$ the authors introduced the idea of fractional conformable integral operators. Jarad et al. [19] introduced the idea of proportional fractional integral operators. Recently, the researchers have established certainly remarkable inequalities, properties, and applications of the fractional conformable integrals and generalized proportional integrals [27, 29, 33-37, 42]. Rahman et al. [37] recently established bounds of proportional fractional integrals for convex functions and their applications.

(c) The Author(s) 2021. This article is licensed under a Creative Commons Attribution 4.0 International License, which permits use, sharing, adaptation, distribution and reproduction in any medium or format, as long as you give appropriate credit to the original author(s) and the source, provide a link to the Creative Commons licence, and indicate if changes were made. The images or other third party material in this article are included in the article's Creative Commons licence, unless indicated otherwise in a credit line to the material. If material is not included in the article's Creative Commons licence and your intended use is not permitted by statutory regulation or exceeds the permitted use, you will need to obtain permission directly from the copyright holder. To view a copy of this licence, visit http://creativecommons.org/licenses/by/4.0/. 
We consider the following extended Chebyshev functional:

$$
\begin{aligned}
\mathfrak{T}(\mathcal{U}, \mathcal{V}, \mu, v)= & \int_{r}^{s} \mu(\vartheta) d \vartheta \int_{r}^{s} v(\vartheta) \mathcal{U}(\vartheta) \mathcal{V}(\vartheta) d \vartheta \\
& +\int_{r}^{s} v(\vartheta) d \vartheta \int_{r}^{s} \mu(\vartheta) \mathcal{U}(\vartheta) \mathcal{V}(\vartheta) d \vartheta \\
& -\left(\int_{r}^{s} v(\vartheta) \mathcal{U}(\vartheta) d \vartheta\right)\left(\int_{r}^{s} \mu(\vartheta) \mathcal{V}(\vartheta) d \vartheta\right) \\
& -\left(\int_{r}^{s} \mu(\vartheta) \mathcal{U}(\vartheta) d \vartheta\right)\left(\int_{r}^{s} v(\vartheta) \mathcal{V}(\vartheta) d \vartheta\right),
\end{aligned}
$$

where the functions $\mathcal{U}$ and $\mathcal{V}$ are integrable on $[r, s]$, and the functions $\mu$ and $\nu$ are positive and integrable on $[r, s]$. The functions $\mathcal{U}$ and $\mathcal{V}$ are said to be synchronous on $[r, s]$ if

$$
(\mathcal{U}(\rho)-\mathcal{U}(\zeta))(\mathcal{V}(\rho)-\mathcal{V}(\zeta)) \geq 0, \quad \rho, \zeta \in[r, s]
$$

The functions $\mathcal{U}$ and $\mathcal{V}$ are said to be asynchronous on $[r, s]$ if the inequality is reversed, that is,

$$
(\mathcal{U}(\rho)-\mathcal{U}(\zeta))(\mathcal{V}(\rho)-\mathcal{V}(\zeta)) \leq 0, \quad \rho, \zeta \in[r, s]
$$

If the functions $\mathcal{U}$ and $\mathcal{V}$ are synchronous on $[r, s]$, then $\mathfrak{T}(\mathcal{U}, \mathcal{V}, \mu, v) \geq 0$. For more detail, see Kuang [23] and Mitrinovic [26]. The Chebyshev functional (1) leads to the Chebyshev inequality [12] if $\mu(\vartheta)=v(\vartheta)=1, \vartheta \in[r, s]$. Ostrowski [30] obtained the following generalization of the Chebyshev inequality: Let $\mathcal{U}$ and $\mathcal{V}$ be differentiable and synchronous functions on $[r, s]$ such that $\left|\mathcal{U}^{\prime}(\vartheta)\right| \geq m$ and $\left|\mathcal{V}^{\prime}(\vartheta)\right| \geq k$ for $\vartheta \in[r, s]$, and let $\mu$ be a positive integrable function on $[r, s]$. Then

$$
\mathfrak{T}(\mathcal{U}, \mathcal{V}, \mu)=\mathfrak{T}(\mathcal{U}, \mathcal{V}, \mu, \nu) \geq m k \mathfrak{T}(\vartheta-a, \vartheta-a ; \mu) \geq 0
$$

If the functions $\mathcal{U}$ and $\mathcal{V}$ are asynchronous on $[r, s]$, then

$$
\mathfrak{T}(\mathcal{U}, \mathcal{V}, \mu)=\mathfrak{T}(\mathcal{U}, \mathcal{V}, \mu, v) \leq m k \mathfrak{T}(\vartheta-a, b-\vartheta ; \mu) \leq 0
$$

If $\mathcal{U}$ and $\mathcal{V}$ are differentiable functions on $[r, s]$ such that $\left|\mathcal{U}^{\prime}(\vartheta)\right| \geq M$ and $\left|\mathcal{V}^{\prime}(\vartheta)\right| \geq K$ for $\vartheta \in[r, s]$ and $\mu$ is a positive integrable function on $[r, s]$, then

$$
|\mathfrak{T}(\mathcal{U}, \mathcal{V}, \mu)| \leq M K \mathfrak{T}(\vartheta-a, \vartheta-a ; \mu) \leq 0
$$

The researchers studied the functional $\mathfrak{T}(\mathcal{U}, \mathcal{V}, \mu)$ and established several extensions and generalizations, which can be found in $[9,10,16,25]$.

\section{Preliminaries}

In this section, we present some well-known definitions and mathematical preliminaries of fractional calculus. 
Definition 2.1 ([31,38]) The Riemann-Liouville (left and right)-sided fractional integrals of order $\kappa>0$ are respectively given by

$$
\left({ }_{r} \mathfrak{J}^{\kappa} \mathcal{U}\right)(x)=\frac{1}{\Gamma(\kappa)} \int_{r}^{\vartheta}(\vartheta-\xi)^{\kappa-1} \mathcal{U}(\xi) d \xi, \quad r<\vartheta
$$

and

$$
\left(\mathfrak{J}_{b}^{\kappa} \mathcal{U}\right)(\vartheta)=\frac{1}{\Gamma(\kappa)} \int_{\vartheta}^{s}(\xi-\vartheta)^{\kappa-1} \mathcal{U}(\xi) d \xi, \quad \vartheta<s
$$

where $\Gamma(\kappa)$ is the classic gamma function.

Definition 2.2 ([19]) The left-sided PFI (proportional fractional integral) is defined by

$$
\left({ }_{r} \mathfrak{J}^{\kappa, \omega} \mathcal{U}\right)(\vartheta)=\frac{1}{\omega^{\kappa} \Gamma(\kappa)} \int_{r}^{\vartheta} \exp \left[\frac{\omega-1}{\omega}(\vartheta-\xi)\right](\vartheta-\xi)^{\kappa-1} \mathcal{U}(\xi) d \xi, \quad r<\vartheta,
$$

where $\kappa>0$ is the order of PFI, and $\omega \in(0,1]$ is the proportionality index.

Definition 2.3 The right-sided PFI (proportional fractional integral) is defined by

$$
\left(\mathfrak{J}_{b}^{\kappa, \omega} \mathcal{U}\right)(x)=\frac{1}{\omega^{\kappa} \Gamma(\kappa)} \int_{\vartheta}^{s} \exp \left[\frac{\omega-1}{\omega}(\xi-\vartheta)\right](\xi-\vartheta)^{\kappa-1} \mathcal{U}(\xi) d \xi, \quad \vartheta<s
$$

Remark 2.1 Setting $\omega=1$ in (4) and (5), we obtain the Riemann-Liouville integrals (2) and (3), respectively.

In this paper, we consider the following one-sided PFI-operator.

Definition 2.4 The one-sided PFI is defined by

$$
\left(\mathfrak{J}_{0}^{\kappa, \omega} \mathcal{U}\right)(\vartheta)=\left(\mathfrak{J}^{\kappa, \omega} \mathcal{U}\right)(\vartheta)=\frac{1}{\omega^{\kappa} \Gamma(\kappa)} \int_{0}^{\vartheta} \exp \left[\frac{\omega-1}{\omega}(\vartheta-\xi)\right](\vartheta-\xi)^{\kappa-1} \mathcal{U}(\xi) d \xi,
$$

where $\kappa>0$ is the order of PFI, and $\omega \in(0,1]$ is the proportionality index.

Definition 2.5 The left-sided Hadamard fractional integral of order $\kappa>0$ is defined by

$$
\left({ }_{r} \mathcal{H}^{\kappa} \mathcal{U}\right)(\vartheta)=\frac{1}{\Gamma(\kappa)} \int_{r}^{\vartheta}(\ln \vartheta-\ln t)^{\kappa-1} \frac{\mathcal{U}(\xi)}{\xi} d \xi, \quad r<\vartheta
$$

Definition 2.6 The right-sided Hadamard fractional integral of order $\kappa>0$ is defined by

$$
\left(\mathcal{H}_{b}^{\kappa} \mathcal{U}\right)(\vartheta)=\frac{1}{\Gamma(\kappa)} \int_{\vartheta}^{s}(\ln \xi-\ln \vartheta)^{\kappa-1} \frac{\mathcal{U}(\xi)}{\xi} d \xi, \quad \vartheta<s .
$$

Definition 2.7 The one-sided Hadamard fractional integral of order $\kappa>0$ is defined by

$$
\left(\mathcal{H}_{1, \vartheta}^{\kappa} \mathcal{U}\right)(\vartheta)=\frac{1}{\Gamma(\kappa)} \int_{1}^{\vartheta}(\ln \vartheta-\ln \xi)^{\kappa-1} \frac{\mathcal{U}(\xi)}{\xi} d \xi, \quad \vartheta>1
$$


Rahman et al. [32] recently presented the following generalized Hadamard proportional fractional integrals.

Definition 2.8 The left-sided Hadamard proportional fractional integral is defined by

$$
\begin{aligned}
& \left({ }_{r} \mathcal{H}^{\kappa, \omega} \mathcal{U}\right)(\vartheta) \\
& \quad=\frac{1}{\omega^{\kappa} \Gamma(\kappa)} \int_{r}^{\vartheta} \exp \left[\frac{\omega-1}{\omega}(\ln \vartheta-\ln \xi)\right](\ln \vartheta-\ln \xi)^{\kappa-1} \frac{\mathcal{U}(\xi)}{\xi} d \xi, \quad r<\vartheta
\end{aligned}
$$

Definition 2.9 The right-sided Hadamard proportional fractional integral is defined by

$$
\begin{aligned}
& \left(\mathcal{H}_{b}^{\kappa, \omega} \mathcal{U}\right)(\vartheta) \\
& =\frac{1}{\omega^{\kappa} \Gamma(\kappa)} \int_{\vartheta}^{s} \exp \left[\frac{\omega-1}{\omega}(\ln \xi-\ln \vartheta)\right](\ln \xi-\ln \vartheta)^{\kappa-1} \frac{\mathcal{U}(\xi)}{\xi} d \xi, \quad \vartheta<s .
\end{aligned}
$$

Definition 2.10 The one-sided Hadamard proportional fractional integral is defined by

$$
\begin{aligned}
& \left(\mathcal{H}_{1, \vartheta}^{\kappa, \omega} \mathcal{U}\right)(\vartheta) \\
& =\frac{1}{\omega^{\kappa} \Gamma(\kappa)} \int_{1}^{\vartheta} \exp \left[\frac{\omega-1}{\omega}(\ln \vartheta-\ln \xi)\right](\ln \vartheta-\ln \xi)^{\kappa-1} \frac{\mathcal{U}(\xi)}{\xi} d \xi, \quad \vartheta>1,
\end{aligned}
$$

where $\kappa>0$ is the order, and $\omega \in(0,1]$ is the proportionality index.

Remark 2.2 Setting $\omega=1,(10)-(12)$ lead to integrals (7)-(9), respectively.

The following results can be easily proved.

\section{Lemma 2.1}

$$
\begin{aligned}
& \left(\mathcal{H}_{1, \vartheta}^{\kappa, \omega} \exp \left[\frac{\omega-1}{\omega}(\ln \vartheta)\right](\ln \vartheta)^{\lambda-1}\right)(\vartheta) \\
& =\frac{\Gamma(\lambda)}{\omega^{\kappa} \Gamma(\kappa+\lambda)} \exp \left[\frac{\omega-1}{\omega}(\ln \vartheta)\right](\ln \vartheta)^{\kappa+\lambda-1},
\end{aligned}
$$

and

$$
\left(\mathcal{H}_{1, \vartheta}^{\kappa, \omega}\right)\left(\mathcal{H}_{1, \vartheta}^{\lambda, \omega}\right) \mathcal{U}(\vartheta)=\left(\mathcal{H}_{1, \vartheta}^{\kappa+\lambda, \omega}\right) \mathcal{U}(\vartheta)
$$

(the semigroup property).

Remark 2.3 Setting $\omega=1,(13)$ reduces to (see [38])

$$
\left(\mathcal{H}_{1, \vartheta}^{\kappa}(\ln \vartheta)^{\lambda-1}\right)(\vartheta)=\frac{\Gamma(\lambda)}{\Gamma(\kappa+\lambda)}(\ln \vartheta)^{\kappa+\lambda-1} .
$$

The paper is organized as follows. In Sect. 3, we present two integral inequalities for the extended Chebyshev functional. The first result concerns one-parameter PFI, and the second one deals with two-parameter PFI. In Sect. 4, we establish integral inequalities for the extended Chebyshev functional by employing the Hadamard proportional fractional integral. 


\section{Certain fractional proportional integral inequalities}

In this section, we present proportional fractional integral inequalities for the extended Chebyshev functional (1) by utilizing the PFI (6). To establish our main result, we first prove the following lemma.

Lemma 3.1 Let $\mathcal{U}$ and $\mathcal{V}$ be synchronous functions on $[0, \infty)$, and let $v, w:[0, \infty) \rightarrow[0, \infty)$. Then for all $\vartheta>0, \kappa>0$, and $\omega \in(0,1]$, we have the following inequality for the PFI-operator (6):

$$
\begin{aligned}
& \mathfrak{J}^{\kappa, \omega}(\nu)(\vartheta) \mathfrak{J}^{\kappa, \omega}(w \mathcal{U} \mathcal{V})(\vartheta)+\mathfrak{J}^{\kappa, \omega}(w)(\vartheta) \mathfrak{J}^{\kappa, \omega}(v \mathcal{U} \mathcal{V})(\vartheta) \\
& \geq \mathfrak{J}^{\kappa, \omega}(\nu \mathcal{U})(\vartheta) \mathfrak{J}^{\kappa, \omega}(w \mathcal{V})(\vartheta)+\mathfrak{J}^{\kappa, \omega}(w \mathcal{U})(\vartheta) \mathfrak{J}^{\kappa, \omega}(\nu \mathcal{V})(\vartheta) .
\end{aligned}
$$

Proof Since the functions $\mathcal{U}$ and $\mathcal{V}$ are synchronous on $[0, \infty)$, for all $\rho \geq 0$ and $\zeta \geq 0$, we have

$$
(\mathcal{U}(\rho)-\mathcal{U}(\zeta))(\mathcal{V}(\rho)-\mathcal{V}(\zeta)) \geq 0
$$

Therefore

$$
\mathcal{U}(\rho) \mathcal{V}(\rho)+\mathcal{U}(\zeta) \mathcal{V}(\zeta) \geq \mathcal{U}(\rho) \mathcal{V}(\zeta)+\mathcal{U}(\zeta) \mathcal{V}(\rho)
$$

Multiplying (17) by $\frac{1}{\omega^{\kappa} \Gamma(\kappa)} e^{\frac{\omega-1}{\omega}(\vartheta-\rho)}(\vartheta-\rho)^{\kappa-1} v(\rho), \rho \in(0, \vartheta)$, and integrating the obtained inequality with respect to $\rho$ over $(0, \vartheta)$, we get

$$
\begin{aligned}
& \frac{1}{\omega^{\kappa} \Gamma(\kappa)} \int_{0}^{\vartheta} e^{\frac{\omega-1}{\omega}(\vartheta-\rho)}(\vartheta-\rho)^{\kappa-1} v(\rho) \mathcal{U}(\rho) \mathcal{V}(\rho) d \rho \\
& \quad+\mathcal{U}(\zeta) \mathcal{V}(\zeta) \frac{1}{\omega^{\kappa} \Gamma(\kappa)} \int_{0}^{\vartheta} e^{\frac{\omega-1}{\omega}(\vartheta-\rho)}(\vartheta-\rho)^{\kappa-1} v(\rho) d \rho \\
& \geq \mathcal{V}(\zeta) \frac{1}{\omega^{\kappa} \Gamma(\kappa)} \int_{0}^{\vartheta} e^{\frac{\omega-1}{\omega}(\vartheta-\rho)}(\vartheta-\rho)^{\kappa-1} v(\rho) \mathcal{U}(\rho) d \rho \\
& \quad+\mathcal{U}(\zeta) \frac{1}{\omega^{\kappa} \Gamma(\kappa)} \int_{0}^{\vartheta} e^{\frac{\omega-1}{\omega}(\vartheta-\rho)}(\vartheta-\rho)^{\kappa-1} v(\rho) \cdot \mathcal{V}(\rho) d \rho
\end{aligned}
$$

In view of (6), we get

$$
\begin{aligned}
& \mathfrak{J}^{\kappa, \omega}(\nu \mathcal{U} \mathcal{V})(\vartheta)+\mathcal{U}(\zeta) \mathcal{V}(\zeta) \mathfrak{J}^{\kappa, \omega}(\nu)(\vartheta) \\
& \quad \geq \mathcal{V}(\zeta) \mathfrak{J}^{\kappa, \omega}(\nu \mathcal{U})(\vartheta)+\mathcal{U}(\zeta) \mathfrak{J}^{\kappa, \omega}(\nu \mathcal{V})(\vartheta) .
\end{aligned}
$$

Now multiplying (18) by $\frac{1}{\omega^{\kappa} \Gamma(\kappa)} e^{\frac{\omega-1}{\omega}(\vartheta-\zeta)}(\vartheta-\zeta)^{\kappa-1} w(\zeta), \zeta \in(0, \vartheta)$, and integrating the obtained inequality with respect to $\zeta$ over $(0, \vartheta)$, we obtain

$$
\begin{aligned}
& \mathfrak{J}^{\kappa, \omega}(v \mathcal{U} \mathcal{V})(\vartheta) \frac{1}{\omega^{\kappa} \Gamma(\kappa)} \int_{0}^{\vartheta} e^{\frac{\omega-1}{\omega}(\vartheta-\zeta)}(\vartheta-\zeta)^{\kappa-1} w(\zeta) d \zeta \\
& \quad+\mathfrak{J}^{\kappa, \omega}(v)(\vartheta) \frac{1}{\omega^{\kappa} \Gamma(\kappa)} \int_{0}^{\vartheta} e^{\frac{\omega-1}{\omega}(\vartheta-\zeta)}(\vartheta-\zeta)^{\kappa-1} w(\zeta) \mathcal{U}(\zeta) \mathcal{V}(\zeta) d \zeta
\end{aligned}
$$




$$
\begin{aligned}
\geq & \mathfrak{J}^{\kappa, \omega}(\nu \mathcal{U})(\vartheta) \frac{1}{\omega^{\kappa} \Gamma(\kappa)} \int_{0}^{\vartheta} e^{\frac{\omega-1}{\omega}(\vartheta-\zeta)}(\vartheta-\zeta)^{\kappa-1} w(\zeta) \mathcal{V}(\zeta) d \zeta \\
& +\mathfrak{J}^{\kappa, \omega}(\nu \mathcal{V})(\vartheta) \frac{1}{\omega^{\kappa} \Gamma(\kappa)} \int_{0}^{\vartheta} e^{\frac{\omega-1}{\omega}(\vartheta-\zeta)}(\vartheta-\zeta)^{\kappa-1} w(\zeta) \mathcal{U}(\zeta) d \zeta
\end{aligned}
$$

which in view of (6) gives the desired inequality (16).

Theorem 3.1 Let $\mathcal{U}$ and $\mathcal{V}$ be synchronous functions on $[0, \infty)$, and let $r, p, q:[0, \infty) \rightarrow$ $[0, \infty)$. Then for all $\vartheta>0, \kappa>0 m$ and $\omega \in(0,1]$, we have the following inequality for the PFI-operator (6):

$$
\begin{aligned}
2 \mathfrak{J}^{\kappa, \omega}(r)(\vartheta)\left[\mathfrak{J}^{\kappa, \omega} p(\vartheta) \mathfrak{J}^{\kappa, \omega}(q \mathcal{U} \mathcal{V})(\vartheta)+\mathfrak{J}^{\kappa, \omega}(q)(\vartheta) \mathfrak{J}^{\kappa, \omega}(p \mathcal{U} \mathcal{V})(\vartheta)\right] \\
\quad+2 \mathfrak{J}^{\kappa, \omega}(p)(\vartheta) \mathfrak{J}^{\kappa, \omega}(q)(\vartheta)(r \mathcal{U})(\vartheta) \\
\geq \mathfrak{J}^{\kappa, \omega}(r)(\vartheta)\left[\mathfrak{J}^{\kappa, \omega}(p \mathcal{U})(\vartheta) \mathfrak{J}^{\kappa, \omega}(q \mathcal{V})(\vartheta)+\mathfrak{J}^{\kappa, \omega}(q \mathcal{U})(\vartheta) \mathfrak{J}^{\kappa, \omega}(p \mathcal{V})(\vartheta)\right] \\
\quad+\mathfrak{J}^{\kappa, \omega}(p)(\vartheta)\left[\mathfrak{J}^{\kappa, \omega}(r \mathcal{U})(\vartheta) \mathfrak{J}^{\kappa, \omega}(q \mathcal{V})(\vartheta)+\mathfrak{J}^{\kappa, \omega}(q \mathcal{U})(\vartheta) \mathfrak{J}^{\kappa, \omega}(r \mathcal{V})(\vartheta)\right] \\
\quad+\mathfrak{J}^{\kappa, \omega}(q)(\vartheta)\left[\mathfrak{J}^{\kappa, \omega}(r \mathcal{U})(\vartheta) \mathfrak{J}^{\kappa, \omega}(p \mathcal{V})(\vartheta)+\mathfrak{J}^{\kappa, \omega}(p \mathcal{U})(\vartheta) \mathfrak{J}^{\kappa, \omega}(r \mathcal{V})(\vartheta)\right] .
\end{aligned}
$$

Proof Taking $v=p$ and $w=q$ in Lemma 3.1, we obtain

$$
\begin{aligned}
& \mathfrak{J}^{\kappa, \omega}(p)(\vartheta) \mathfrak{J}^{\kappa, \omega}(q \mathcal{U} \mathcal{V})(\vartheta)+\mathfrak{J}^{\kappa, \omega}(q)(\vartheta) \mathfrak{J}^{\kappa, \omega}(p \mathcal{U} \mathcal{V})(\vartheta) \\
& \geq \mathfrak{J}^{\kappa, \omega}(p \mathcal{U})(\vartheta) \mathfrak{J}^{\kappa, \omega}(q \mathcal{V})(\vartheta)+\mathfrak{J}^{\kappa, \omega}(q \mathcal{U})(\vartheta) \mathfrak{J}^{\kappa, \omega}(p \mathcal{V})(\vartheta) .
\end{aligned}
$$

Multiplying both sides of (21) by $\mathfrak{J}^{\kappa, \omega}(r)(\vartheta)$, we get

$$
\begin{aligned}
& \mathfrak{J}^{\kappa, \omega}(r)(\vartheta)\left[\mathfrak{J}^{\kappa, \omega}(p)(\vartheta) \mathfrak{J}^{\kappa, \omega}(q \mathcal{U} \mathcal{V})(\vartheta)+\mathfrak{J}^{\kappa, \omega}(q)(\vartheta) \mathfrak{J}^{\kappa, \omega}(p \mathcal{U} \mathcal{V})(\vartheta)\right] \\
& \geq \mathfrak{J}^{\kappa, \omega}(r)(\vartheta)\left[\mathfrak{J}^{\kappa, \omega}(p \mathcal{U})(\vartheta) \mathfrak{J}^{\kappa, \omega}(q \mathcal{V})(\vartheta)+\mathfrak{J}^{\kappa, \omega}(q \mathcal{U})(\vartheta) \mathfrak{J}^{\kappa, \omega}(p \mathcal{V})(\vartheta)\right] .
\end{aligned}
$$

Now taking $v=r$ and $w=q$ in Lemma 3.1, we have

$$
\begin{aligned}
& \mathfrak{J}^{\kappa, \omega}(r)(\vartheta) \mathfrak{J}^{\kappa, \omega}(q \mathcal{U} \mathcal{V})(\vartheta)+\mathfrak{J}^{\kappa, \omega}(q)(\vartheta) \mathfrak{J}^{\kappa, \omega}(r \mathcal{U} \mathcal{V})(\vartheta) \\
& \geq \mathfrak{J}^{\kappa, \omega}(r \mathcal{U})(\vartheta) \mathfrak{J}^{\kappa, \omega}(q \mathcal{V})(\vartheta)+\mathfrak{J}^{\kappa, \omega}(q \mathcal{U})(\vartheta) \mathfrak{J}^{\kappa, \omega}(r \mathcal{V})(\vartheta) .
\end{aligned}
$$

Multiplying both sides of (23) by $\mathfrak{J}^{\kappa, \omega}(p)(\vartheta)$, we get

$$
\begin{aligned}
& \mathfrak{J}^{\kappa, \omega}(p)(\vartheta)\left[\mathfrak{J}^{\kappa, \omega}(r)(\vartheta) \mathfrak{J}^{\kappa, \omega}(q \mathcal{U} \mathcal{V})(\vartheta)+\mathfrak{J}^{\kappa, \omega}(q)(\vartheta) \mathfrak{J}^{\kappa, \omega}(r \mathcal{U} \mathcal{V})(\vartheta)\right] \\
& \geq \mathfrak{J}^{\kappa, \omega}(p)(\vartheta)\left[\mathfrak{J}^{\kappa, \omega}(r \mathcal{U})(\vartheta) \mathfrak{J}^{\kappa, \omega}(q \mathcal{V})(\vartheta)+\mathfrak{J}^{\kappa, \omega}(q \mathcal{U})(\vartheta) \mathfrak{J}^{\kappa, \omega}(r \mathcal{V})(\vartheta)\right] .
\end{aligned}
$$

Similarly, taking $v=r$ and $w=p$ in Lemma 3.1 and then multiplying both sides of the resultant inequality by $\mathfrak{J}^{\kappa, \omega}(q)(\vartheta)$, we obtain

$$
\begin{aligned}
& \mathfrak{J}^{\kappa, \omega}(q)(\vartheta)\left[\mathfrak{J}^{\kappa, \omega}(r)(\vartheta) \mathfrak{J}^{\kappa, \omega}(p \mathcal{U} \mathcal{V})(\vartheta)+\mathfrak{J}^{\kappa, \omega}(p)(\vartheta) \mathfrak{J}^{\kappa, \omega}(r \mathcal{U} \mathcal{V})(\vartheta)\right] \\
& \geq \mathfrak{J}^{\kappa, \omega}(q)(\vartheta)\left[\mathfrak{J}^{\kappa, \omega}(r \mathcal{U})(\vartheta) \mathfrak{J}^{\kappa, \omega}(p \mathcal{V})(\vartheta)+\mathfrak{J}^{\kappa, \omega}(p \mathcal{U})(\vartheta) \mathfrak{J}^{\kappa, \omega}(r \mathcal{V})(\vartheta)\right] .
\end{aligned}
$$

Hence by adding (22), (24), and (25) we get the desired statement (20). 
Remark 3.1 Setting $\omega=1$ in Theorem 3.1, we get Theorem 2 proved by Dahmani [14].

Lemma 3.2 Let $\mathcal{U}$ and $\mathcal{V}$ be synchronous functions on $[0, \infty)$, and let $v, w:[0, \infty) \rightarrow[0, \infty)$. Then for all $\vartheta>0, \kappa, \eta>0$, and $\omega \in(0,1]$, we have the following inequality for the PFIoperator (6):

$$
\begin{aligned}
& \mathfrak{J}^{\kappa, \omega}(\nu)(\vartheta) \mathfrak{J}^{\eta, \omega}(w \mathcal{U} \mathcal{V})(\vartheta)+\mathfrak{J}^{\eta, \omega}(w)(\vartheta) \mathfrak{J}^{\kappa, \omega}(\nu \mathcal{U} \mathcal{V})(\vartheta) \\
& \geq \mathfrak{J}^{\kappa, \omega}(\nu \mathcal{U})(\vartheta) \mathfrak{J}^{\eta, \omega}(w \mathcal{V})(\vartheta)+\mathfrak{J}^{\eta, \omega}(w \mathcal{U})(\vartheta) \mathfrak{J}^{\kappa, \omega}(\nu \mathcal{V})(\vartheta) .
\end{aligned}
$$

Proof Multiplying (19) by $\frac{1}{\omega^{\eta} \Gamma(\eta)} e^{\frac{\omega-1}{\omega}(\vartheta-\zeta)}(\vartheta-\zeta)^{\eta-1} w(\zeta), \zeta \in(0, \vartheta)$ and integrating the obtained inequality with respect to $\zeta$ over $(0, \vartheta)$, we obtain

$$
\begin{aligned}
& \mathfrak{J}^{\kappa, \omega}(v \mathcal{U} \mathcal{V})(\vartheta) \frac{1}{\omega^{\eta} \Gamma(\eta)} \int_{0}^{\vartheta} e^{\frac{\omega-1}{\omega}(\vartheta-\zeta)}(\vartheta-\zeta)^{\eta-1} w(\zeta) d \zeta \\
& \quad+\mathfrak{J}^{\kappa, \omega}(v)(\vartheta) \frac{1}{\omega^{\eta} \Gamma(\eta)} \int_{0}^{\vartheta} e^{\frac{\omega-1}{\omega}(\vartheta-\zeta)}(\vartheta-\zeta)^{\eta-1} w(\zeta) \mathcal{U}(\zeta) \mathcal{V}(\zeta) d \zeta \\
& \geq \mathfrak{J}^{\kappa, \omega}(\nu \mathcal{U})(\vartheta) \frac{1}{\omega^{\eta} \Gamma(\eta)} \int_{0}^{\vartheta} e^{\frac{\omega-1}{\omega}(\vartheta-\zeta)}(\vartheta-\zeta)^{\eta-1} w(\zeta) \mathcal{V}(\zeta) d \zeta \\
& \quad+\mathfrak{J}^{\kappa, \omega}(v \mathcal{V})(\vartheta) \frac{1}{\omega^{\eta} \Gamma(\eta)} \int_{0}^{\vartheta} e^{\frac{\omega-1}{\omega}(\vartheta-\zeta)}(\vartheta-\zeta)^{\eta-1} w(\zeta) \mathcal{U}(\zeta) d \zeta,
\end{aligned}
$$

which by (6) gives

$$
\begin{aligned}
& \mathfrak{J}^{\kappa, \omega}(v)(\vartheta) \mathfrak{J}^{\eta, \omega}(w \mathcal{U} \mathcal{V})(\vartheta)+\mathfrak{J}^{\eta, \omega}(w)(\vartheta) \mathfrak{J}^{\kappa, \omega}(v \mathcal{U} \mathcal{V})(\vartheta) \\
& \geq \mathfrak{J}^{\kappa, \omega}(\nu \mathcal{U})(\vartheta) \mathfrak{J}^{\eta, \omega}(w \mathcal{V})(\vartheta)+\mathfrak{J}^{\eta, \omega}(w \mathcal{U})(\vartheta) \mathfrak{J}^{\kappa, \omega}(\nu \mathcal{V})(\vartheta) .
\end{aligned}
$$

This completes the proof of Lemma 3.2.

Remark 3.2 Setting $\kappa=\eta$ in Lemma 3.2, we get Lemma 3.1.

Theorem 3.2 Let $\mathcal{U}$ and $\mathcal{V}$ be synchronous functions on $[0, \infty)$, and let $r, p, q:[0, \infty) \rightarrow$ $[0, \infty)$. Then for all $\vartheta>0, \kappa>0 m$ and $\omega \in(0,1]$, we have the following inequality for the PFI-operator (6):

$$
\begin{aligned}
\mathfrak{J}^{\kappa, \omega}(r)(\vartheta)\left[\mathfrak{J}^{\kappa, \omega} p(\vartheta) \mathfrak{J}^{\eta, \omega}(q \mathcal{U} \mathcal{V})(\vartheta)+2 \mathfrak{J}^{\kappa, \omega}(p)(\vartheta) \mathfrak{J}^{\eta, \omega}(q \mathcal{U} \mathcal{V})(\vartheta)\right. \\
+ \\
\left.+\mathfrak{J}^{\eta, \omega}(q)(\vartheta) \mathfrak{J}^{\kappa, \omega}(p \mathcal{U} \mathcal{V})(\vartheta)\right] \\
+\left[\mathfrak{J}^{\kappa, \omega}(p)(\vartheta) \mathfrak{J}^{\eta, \omega}(q)(\vartheta)+\mathfrak{J}^{\eta, \omega}(p)(\vartheta) \mathfrak{J}^{\kappa, \omega}(q)(\vartheta)\right] \mathfrak{J}^{\kappa, \omega}(r \mathcal{U} \mathcal{V})(\vartheta) \\
\geq \mathfrak{J}^{\kappa, \omega}(r)(\vartheta)\left[\mathfrak{J}^{\kappa, \omega}(p \mathcal{U})(\vartheta) \mathfrak{J}^{\eta, \omega}(q \mathcal{V})(\vartheta)+\mathfrak{J}^{\eta, \omega}(q \mathcal{U})(\vartheta) \mathfrak{J}^{\kappa, \omega}(p \mathcal{V})(\vartheta)\right] \\
+\mathfrak{J}^{\kappa, \omega}(p)(\vartheta)\left[\mathfrak{J}^{\kappa, \omega}(r \mathcal{U})(\vartheta) \mathfrak{J}^{\eta, \omega}(q \mathcal{V})(\vartheta)+\mathfrak{J}^{\eta, \omega}(q \mathcal{U})(\vartheta) \mathfrak{J}^{\kappa, \omega}(r \mathcal{V})(\vartheta)\right] \\
+\mathfrak{J}^{\kappa, \omega}(q)(\vartheta)\left[\mathfrak{J}^{\kappa, \omega}(r \mathcal{U})(\vartheta) \mathfrak{J}^{\eta, \omega}(p \mathcal{V})(\vartheta)+\mathfrak{J}^{\eta, \omega}(p \mathcal{U})(\vartheta) \mathfrak{J}^{\kappa, \omega}(r \mathcal{V})(\vartheta)\right] .
\end{aligned}
$$


Proof Taking $v=p$ and $w=q$ in Lemma 3.2, we obtain

$$
\begin{aligned}
\mathfrak{J}^{\kappa, \omega}(p)(\vartheta) \mathfrak{J}^{\eta, \omega}(q \mathcal{U} \mathcal{V})(\vartheta)+\mathfrak{J}^{\eta, \omega}(q)(\vartheta) \mathfrak{J}^{\kappa, \omega}(p \mathcal{U} \mathcal{V})(\vartheta) \\
\geq \mathfrak{J}^{\kappa, \omega}(p \mathcal{U})(\vartheta) \mathfrak{J}^{\eta, \omega}(q \mathcal{V})(\vartheta)+\mathfrak{J}^{\eta, \omega}(q \mathcal{U})(\vartheta) \mathfrak{J}^{\kappa, \omega}(p \mathcal{V})(\vartheta)
\end{aligned}
$$

Multiplying (28) by $\mathfrak{J}^{\kappa, \omega}(r)(\vartheta)$, we get

$$
\begin{aligned}
& \mathfrak{J}^{\kappa, \omega}(r)(\vartheta)\left[\mathfrak{J}^{\kappa, \omega}(p)(\vartheta) \mathfrak{J}^{\eta, \omega}(q \mathcal{U} \mathcal{V})(\vartheta)+\mathfrak{J}^{\eta, \omega}(q)(\vartheta) \mathfrak{J}^{\kappa, \omega}(p \mathcal{U} \mathcal{V})(\vartheta)\right] \\
& \geq \mathfrak{J}^{\kappa, \omega}(r)(\vartheta)\left[\mathfrak{J}^{\kappa, \omega}(p \mathcal{U})(\vartheta) \mathfrak{J}^{\eta, \omega}(q \mathcal{V})(\vartheta)+\mathfrak{J}^{\eta, \omega}(q \mathcal{U})(\vartheta) \mathfrak{J}^{\kappa, \omega}(p \mathcal{V})(\vartheta)\right]
\end{aligned}
$$

Setting $v=r$ and $w=q$ in Lemma 3.2, we obtain

$$
\begin{aligned}
& \mathfrak{J}^{\kappa, \omega}(r)(\vartheta) \mathfrak{J}^{\eta, \omega}(q \mathcal{U} \mathcal{V})(\vartheta)+\mathfrak{J}^{\eta, \omega}(q)(\vartheta) \mathfrak{J}^{\kappa, \omega}(r \mathcal{U} \mathcal{V})(\vartheta) \\
& \geq \mathfrak{J}^{\kappa, \omega}(r \mathcal{U})(\vartheta) \mathfrak{J}^{\eta, \omega}(q \mathcal{V})(\vartheta)+\mathfrak{J}^{\eta, \omega}(q \mathcal{U})(\vartheta) \mathfrak{J}^{\kappa, \omega}(r \mathcal{V})(\vartheta) .
\end{aligned}
$$

Multiplying (30) by $\mathfrak{J}^{\kappa, \omega}(p)(\vartheta)$, we get

$$
\begin{aligned}
\mathfrak{J}^{\kappa, \omega}(p)(\vartheta)\left[\mathfrak{J}^{\kappa, \omega}(r)(\vartheta) \mathfrak{J}^{\eta, \omega}(q \mathcal{U} \mathcal{V})(\vartheta)+\mathfrak{J}^{\eta, \omega}(q)(\vartheta) \mathfrak{J}^{\kappa, \omega}(r \mathcal{U} \mathcal{V})(\vartheta)\right] \\
\geq \mathfrak{J}^{\kappa, \omega}(p)(\vartheta)\left[\mathfrak{J}^{\kappa, \omega}(r \mathcal{U})(\vartheta) \mathfrak{J}^{\eta, \omega}(q \mathcal{V})(\vartheta)+\mathfrak{J}^{\eta, \omega}(q \mathcal{U})(\vartheta) \mathfrak{J}^{\kappa, \omega}(r \mathcal{V})(\vartheta)\right] .
\end{aligned}
$$

Similarly, setting $v=r$ and $w=p$ in Lemma 3.2 and multiplying both sides of the resultant inequality by $\mathfrak{J}^{\kappa, \omega}(q)(\vartheta)$, we obtain

$$
\begin{aligned}
& \mathfrak{J}^{\kappa, \omega}(q)(\vartheta)\left[\mathfrak{J}^{\kappa, \omega}(r)(\vartheta) \mathfrak{J}^{\eta, \omega}(p \mathcal{U} \mathcal{V})(\vartheta)+\mathfrak{J}^{\eta, \omega}(p)(\vartheta) \mathfrak{J}^{\kappa, \omega}(r \mathcal{U} \mathcal{V})(\vartheta)\right] \\
& \quad \geq \mathfrak{J}^{\kappa, \omega}(q)(\vartheta)\left[\mathfrak{J}^{\kappa, \omega}(r \mathcal{U})(\vartheta) \mathfrak{J}^{\eta, \omega}(p \mathcal{V})(\vartheta)+\mathfrak{J}^{\eta, \omega}(p \mathcal{U})(\vartheta) \mathfrak{J}^{\kappa, \omega}(r \mathcal{V})(\vartheta)\right]
\end{aligned}
$$

Hence we obtain statement (27) by adding inequalities (29), (31), and (32).

Remark 3.3 Setting $\kappa=\eta$ in Theorem 3.2, we get Theorem 3.1.

Remark 3.4 Setting $\omega=1$ in Theorem 3.2, we get Theorem 4 proved by Dahmani [14].

Remark 3.5 Inequalities (20) and (27) will be reversed in the following cases:

(i) The functions $\mathcal{U}$ and $\mathcal{V}$ are asynchronous on $[0, \infty)$.

(ii) The functions $r, p$, and $q$ are negative on $[0, \infty)$.

(iii) Two of the functions $r, p$, and $q$ are positive, and the third one is negative on $[0, \infty)$.

Remark 3.6 The Chebyshev inequality [12] on $[0, x]$ can be obtained for any $\vartheta \in[0, \infty)$ if we set $\kappa=\eta=\omega=1$ and $p(\vartheta)=q(\vartheta)=r(\vartheta)=1$ in Theorem 3.2.

\section{Inequalities via Hadamard proportional fractional integral}

In this section, we present some inequalities for the extended Chebyshev functional (1) by employing the Hadamard proportional fractional integral (12). 
Lemma 4.1 Let $\mathcal{U}$ and $\mathcal{V}$ be synchronous functions on $[0, \infty)$, and let $v, w:[0, \infty) \rightarrow[0, \infty)$. Then for all $\vartheta>1, \kappa>0$, and $\omega \in(0,1]$, we have the following inequality for (12):

$$
\begin{aligned}
& \mathcal{H}_{1, \vartheta}^{\kappa, \omega}(v)(\vartheta) \mathcal{H}_{1, \vartheta}^{\kappa, \omega}(w \mathcal{U} \mathcal{V})(\vartheta)+\mathcal{H}_{1, \vartheta}^{\kappa, \omega}(w)(\vartheta) \mathcal{H}_{1, \vartheta}^{\kappa, \omega}(v \mathcal{U} \mathcal{V})(\vartheta) \\
& \geq \mathcal{H}_{1, \vartheta}^{\kappa, \omega}(\nu \mathcal{U})(\vartheta) \mathcal{H}_{1, \vartheta}^{\kappa, \omega}(w \mathcal{V})(\vartheta)+\mathcal{H}_{1, \vartheta}^{\kappa, \omega}(w \mathcal{U})(\vartheta) \mathcal{H}_{1, \vartheta}^{\kappa, \omega}(\nu \mathcal{V})(\vartheta)
\end{aligned}
$$

Proof Since the functions $\mathcal{U}$ and $\mathcal{V}$ are synchronous on $[0, \infty)$, for all $\rho \geq 0, \zeta \geq 0$, we have

$$
(\mathcal{U}(\rho)-\mathcal{U}(\zeta))(\mathcal{V}(\rho)-\mathcal{V}(\zeta)) \geq 0
$$

Therefore

$$
\mathcal{U}(\rho) \mathcal{V}(\rho)+\mathcal{U}(\zeta) \mathcal{V}(\zeta) \geq \mathcal{U}(\rho) \mathcal{V}(\zeta)+\mathcal{U}(\zeta) \mathcal{V}(\rho)
$$

Multiplying (34) by $\frac{1}{\omega^{\kappa} \Gamma(\kappa)} e^{\frac{\omega-1}{\omega}(\ln \vartheta-\ln \rho)}(\ln \vartheta-\ln \rho)^{\kappa-1} \frac{v(\rho)}{\rho}, \rho \in(1, \vartheta)$, and integrating the obtained inequality with respect to $\rho$ over $(1, \vartheta)$, we get

$$
\begin{aligned}
& \frac{1}{\omega^{\kappa} \Gamma(\kappa)} \int_{1}^{\vartheta} e^{\frac{\omega-1}{\omega}(\ln \vartheta-\ln \rho)}(\ln \vartheta-\ln \rho)^{\kappa-1} v(\rho) \mathcal{U}(\rho) \mathcal{V}(\rho) \frac{d \rho}{\rho} \\
& \quad+\mathcal{U}(\zeta) \mathcal{V}(\zeta) \frac{1}{\omega^{\kappa} \Gamma(\kappa)} \int_{1}^{\vartheta} e^{\frac{\omega-1}{\omega}(\ln \vartheta-\ln \rho)}(\ln \vartheta-\ln \rho)^{\kappa-1} v(\rho) \frac{d \rho}{\rho} \\
& \geq \\
& \quad \mathcal{V}(\zeta) \frac{1}{\omega^{\kappa} \Gamma(\kappa)} \int_{1}^{\vartheta} e^{\frac{\omega-1}{\omega}(\ln \vartheta-\ln \rho)}(\ln \vartheta-\ln \rho)^{\kappa-1} v(\rho) \mathcal{U}(\rho) \frac{d \rho}{\rho} \\
& \quad+\mathcal{U}(\zeta) \frac{1}{\omega^{\kappa} \Gamma(\kappa)} \int_{1}^{\vartheta} e^{\frac{\omega-1}{\omega}(\ln \vartheta-\ln \rho)}(\ln \vartheta-\ln \rho)^{\kappa-1} v(\rho) \mathcal{V}(\rho) \frac{d \rho}{\rho}
\end{aligned}
$$

In view of (12), we get

$$
\begin{aligned}
& \mathcal{H}_{1, \vartheta}^{\kappa, \omega}(\nu \mathcal{U} \mathcal{V})(\vartheta)+\mathcal{U}(\zeta) \mathcal{V}(\zeta) \mathcal{H}_{1, \vartheta}^{\kappa, \omega}(v)(\vartheta) \\
& \quad \geq \mathcal{V}(\zeta) \mathcal{H}_{1, \vartheta}^{\kappa, \omega}(\nu \mathcal{U})(\vartheta)+\mathcal{U}(\zeta) \mathcal{H}_{1, \vartheta}^{\kappa, \omega}(\nu \mathcal{V})(\vartheta)
\end{aligned}
$$

Now multiplying (35) by $\frac{1}{\omega^{\kappa} \Gamma(\kappa)} e^{\frac{\omega-1}{\omega}(\ln \vartheta-\ln \zeta)}(\ln \vartheta-\ln \zeta)^{\kappa-1} \frac{w(\zeta)}{\zeta}, \zeta \in(1, \vartheta)$, and integrating the obtained inequality with respect to $\zeta$ over $(1, \vartheta)$, we obtain

$$
\begin{aligned}
\mathcal{H}_{1, \vartheta}^{\kappa, \omega} & (\mathcal{U} \mathcal{V})(\vartheta) \frac{1}{\omega^{\kappa} \Gamma(\kappa)} \int_{1}^{\vartheta} e^{\frac{\omega-1}{\omega}(\ln \vartheta-\ln \zeta)}(\ln \vartheta-\ln \zeta)^{\kappa-1} w(\zeta) \frac{d \zeta}{\zeta} \\
& +\mathcal{H}_{1, \vartheta}^{\kappa, \omega}(\nu)(\vartheta) \frac{1}{\omega^{\kappa} \Gamma(\kappa)} \int_{1}^{\vartheta} e^{\frac{\omega-1}{\omega}(\ln \vartheta-\ln \zeta)}(\ln \vartheta-\ln \zeta)^{\kappa-1} w(\zeta) \mathcal{U}(\zeta) \mathcal{V}(\zeta) \frac{d \zeta}{\zeta} \\
\geq & \mathcal{H}_{1, \vartheta}^{\kappa, \omega}(\mathcal{U U})(\vartheta) \frac{1}{\omega^{\kappa} \Gamma(\kappa)} \int_{1}^{\vartheta} e^{\frac{\omega-1}{\omega}(\ln \vartheta-\ln \zeta)}(\ln \vartheta-\ln \zeta)^{\kappa-1} w(\zeta) \mathcal{V}(\zeta) \frac{d \zeta}{\zeta} \\
& +\mathcal{H}_{1, \vartheta}^{\kappa, \omega}(\nu \mathcal{V})(\vartheta) \frac{1}{\omega^{\kappa} \Gamma(\kappa)} \int_{1}^{\vartheta} e^{\frac{\omega-1}{\omega}(\ln \vartheta-\ln \zeta)}(\ln \vartheta-\ln \zeta)^{\kappa-1} w(\zeta) \mathcal{U}(\zeta) \frac{d \zeta}{\zeta}
\end{aligned}
$$

which in view of (6) gives the desired inequality (16). 
Theorem 4.1 Let $\mathcal{U}$ and $\mathcal{V}$ be synchronous functions on $[0, \infty)$, and let $r, p, q:[0, \infty) \rightarrow$ $[0, \infty)$. Then for all $\vartheta>1, \kappa>0$, and $\omega \in(0,1]$, we have the following inequality for (12):

$$
\begin{aligned}
2 \mathcal{H}_{1, \vartheta}^{\kappa, \omega}(r)(\vartheta)\left[\mathcal{H}_{1, \vartheta}^{\kappa, \omega} p(\vartheta) \mathcal{H}_{1, \vartheta}^{\kappa, \omega}(q \mathcal{U} \mathcal{V})(\vartheta)+\mathcal{H}_{1, \vartheta}^{\kappa, \omega}(q)(\vartheta) \mathcal{H}_{1, \vartheta}^{\kappa, \omega}(p \mathcal{U} \mathcal{V})(\vartheta)\right] \\
+2 \mathcal{H}_{1, \vartheta}^{\kappa, \omega}(p)(\vartheta) \mathcal{H}_{1, \vartheta}^{\kappa, \omega}(q)(\vartheta)(r \mathcal{U})(\vartheta) \\
\geq \mathcal{H}_{1, \vartheta}^{\kappa, \omega}(r)(\vartheta)\left[\mathcal{H}_{1, \vartheta}^{\kappa, \omega}(p \mathcal{U})(\vartheta) \mathcal{H}_{1, \vartheta}^{\kappa, \omega}(q \mathcal{V})(\vartheta)+\mathcal{H}_{1, \vartheta}^{\kappa, \omega}(q \mathcal{U})(\vartheta) \mathcal{H}_{1, \vartheta}^{\kappa, \omega}(p \mathcal{V})(\vartheta)\right] \\
+\mathcal{H}_{1, \vartheta}^{\kappa, \omega}(p)(\vartheta)\left[\mathcal{H}_{1, \vartheta}^{\kappa, \omega}(r \mathcal{U})(\vartheta) \mathcal{H}_{1, \vartheta}^{\kappa, \omega}(q \mathcal{V})(\vartheta)+\mathcal{H}_{1, \vartheta}^{\kappa, \omega}(q \mathcal{U})(\vartheta) \mathcal{H}_{1, \vartheta}^{\kappa, \omega}(r \mathcal{V})(\vartheta)\right] \\
+\mathcal{H}_{1, \vartheta}^{\kappa, \omega}(q)(\vartheta)\left[\mathcal{H}_{1, \vartheta}^{\kappa, \omega}(r \mathcal{U})(\vartheta) \mathcal{H}_{1, \vartheta}^{\kappa, \omega}(p \mathcal{V})(\vartheta)+\mathcal{H}_{1, \vartheta}^{\kappa, \omega}(p \mathcal{U})(\vartheta) \mathcal{H}_{1, \vartheta}^{\kappa, \omega}(r \mathcal{V})(\vartheta)\right] .
\end{aligned}
$$

Proof Taking $v=p$ and $w=q$ in Lemma 4.1, we obtain

$$
\begin{aligned}
& \mathcal{H}_{1, \vartheta}^{\kappa, \omega}(p)(\vartheta) \mathcal{H}_{1, \vartheta}^{\kappa, \omega}(q \mathcal{U} \mathcal{V})(\vartheta)+\mathcal{H}_{1, \vartheta}^{\kappa, \omega}(q)(\vartheta) \mathcal{H}_{1, \vartheta}^{\kappa, \omega}(p \mathcal{U} \mathcal{V})(\vartheta) \\
& \geq \mathcal{H}_{1, \vartheta}^{\kappa, \omega}(p \mathcal{U})(\vartheta) \mathcal{H}_{1, \vartheta}^{\kappa, \omega}(q \mathcal{V})(\vartheta)+\mathcal{H}_{1, \vartheta}^{\kappa, \omega}(q \mathcal{U})(\vartheta) \mathcal{H}_{1, \vartheta}^{\kappa, \omega}(p \mathcal{V})(\vartheta) .
\end{aligned}
$$

Multiplying (37) by $\mathcal{H}_{1, \vartheta}^{\kappa, \omega}(r)(\vartheta)$, we get

$$
\begin{aligned}
& \mathcal{H}_{1, \vartheta}^{\kappa, \omega}(r)(\vartheta)\left[\mathcal{H}_{1, \vartheta}^{\kappa, \omega}(p)(\vartheta) \mathcal{H}_{1, \vartheta}^{\kappa, \omega}(q \mathcal{U} \mathcal{V})(\vartheta)+\mathcal{H}_{1, \vartheta}^{\kappa, \omega}(q)(\vartheta) \mathcal{H}_{1, \vartheta}^{\kappa, \omega}(p \mathcal{U} \mathcal{V})(\vartheta)\right] \\
& \geq \mathcal{H}_{1, \vartheta}^{\kappa, \omega}(r)(\vartheta)\left[\mathcal{H}_{1, \vartheta}^{\kappa, \omega}(p \mathcal{U})(\vartheta) \mathcal{H}_{1, \vartheta}^{\kappa, \omega}(q \mathcal{V})(\vartheta)+\mathcal{H}_{1, \vartheta}^{\kappa, \omega}(q \mathcal{U})(\vartheta) \mathcal{H}_{1, \vartheta}^{\kappa, \omega}(p \mathcal{V})(\vartheta)\right] .
\end{aligned}
$$

Now taking $v=r$ and $w=q$ in Lemma 4.1, we have

$$
\begin{aligned}
& \mathcal{H}_{1, \vartheta}^{\kappa, \omega}(r)(\vartheta) \mathcal{H}_{1, \vartheta}^{\kappa, \omega}(q \mathcal{U} \mathcal{V})(\vartheta)+\mathcal{H}_{1, \vartheta}^{\kappa, \omega}(q)(\vartheta) \mathcal{H}_{1, \vartheta}^{\kappa, \omega}(r \mathcal{U} \mathcal{V})(\vartheta) \\
& \geq \mathcal{H}_{1, \vartheta}^{\kappa, \omega}(r \mathcal{U})(\vartheta) \mathcal{H}_{1, \vartheta}^{\kappa, \omega}(q \mathcal{V})(\vartheta)+\mathcal{H}_{1, \vartheta}^{\kappa, \omega}(q \mathcal{U})(\vartheta) \mathcal{H}_{1, \vartheta}^{\kappa, \omega}(r \mathcal{V})(\vartheta) .
\end{aligned}
$$

Multiplying (39) by $\mathcal{H}_{1, \vartheta}^{\kappa, \omega}(p)(\vartheta)$, we get

$$
\begin{aligned}
\mathcal{H}_{1, \vartheta}^{\kappa, \omega}(p)(\vartheta)\left[\mathcal{H}_{1, \vartheta}^{\kappa, \omega}(r)(\vartheta) \mathcal{H}_{1, \vartheta}^{\kappa, \omega}(q \mathcal{U} \mathcal{V})(\vartheta)+\mathcal{H}_{1, \vartheta}^{\kappa, \omega}(q)(\vartheta) \mathcal{H}_{1, \vartheta}^{\kappa, \omega}(r \mathcal{U} \mathcal{V})(\vartheta)\right] \\
\geq \mathcal{H}_{1, \vartheta}^{\kappa, \omega}(p)(\vartheta)\left[\mathcal{H}_{1, \vartheta}^{\kappa, \omega}(r \mathcal{U})(\vartheta) \mathcal{H}_{1, \vartheta}^{\kappa, \omega}(q \mathcal{V})(\vartheta)+\mathcal{H}_{1, \vartheta}^{\kappa, \omega}(q \mathcal{U})(\vartheta) \mathcal{H}_{1, \vartheta}^{\kappa, \omega}(r \mathcal{V})(\vartheta)\right] .
\end{aligned}
$$

Similarly, taking $v=r$ and $w=p$ in Lemma 4.1 and then multiplying both sides of the resultant inequality by $\mathcal{H}_{1, \vartheta}^{\kappa, \omega}(q)(\vartheta)$, we obtain

$$
\begin{aligned}
\mathcal{H}_{1, \vartheta}^{\kappa, \omega}(q)(\vartheta)\left[\mathcal{H}_{1, \vartheta}^{\kappa, \omega}(r)(\vartheta) \mathcal{H}_{1, \vartheta}^{\kappa, \omega}(p \mathcal{U} \mathcal{V})(\vartheta)+\mathcal{H}_{1, \vartheta}^{\kappa, \omega}(p)(\vartheta) \mathcal{H}_{1, \vartheta}^{\kappa, \omega}(r \mathcal{U} \mathcal{V})(\vartheta)\right] \\
\geq \mathcal{H}_{1, \vartheta}^{\kappa, \omega}(q)(\vartheta)\left[\mathcal{H}_{1, \vartheta}^{\kappa, \omega}(r \mathcal{U})(\vartheta) \mathcal{H}_{1, \vartheta}^{\kappa, \omega}(p \mathcal{V})(\vartheta)+\mathcal{H}_{1, \vartheta}^{\kappa, \omega}(p \mathcal{U})(\vartheta) \mathcal{H}_{1, \vartheta}^{\kappa, \omega}(r \mathcal{V})(\vartheta)\right] .
\end{aligned}
$$

Hence by adding (38), (40), and (41) we get the desired statement (36).

Remark 4.1 Setting $\omega=1$ in Theorem 4.1, we get Theorem 3.2 proved by Chinchane and Pachpatte [13]. 
Lemma 4.2 Let $\mathcal{U}$ and $\mathcal{V}$ be synchronous functions on $[0, \infty)$, and let $v, w:[0, \infty) \rightarrow[0, \infty)$. Then for all $\vartheta>1, \kappa, \eta>0$, and $\omega \in(0,1]$, we have the following inequality for (12):

$$
\begin{aligned}
& \mathcal{H}_{1, \vartheta}^{\kappa, \omega}(\nu)(\vartheta) \mathcal{H}_{1, \vartheta}^{\eta, \omega}(w \mathcal{U} \mathcal{V})(\vartheta)+\mathcal{H}_{1, \vartheta}^{\eta, \omega}(w)(\vartheta) \mathcal{H}_{1, \vartheta}^{\kappa, \omega}(\nu \mathcal{U} \mathcal{V})(\vartheta) \\
& \geq \mathcal{H}_{1, \vartheta}^{\kappa, \omega}(\nu \mathcal{U})(\vartheta) \mathcal{H}_{1, \vartheta}^{\eta, \omega}(w \mathcal{V})(\vartheta)+\mathcal{H}_{1, \vartheta}^{\eta, \omega}(w \mathcal{U})(\vartheta) \mathcal{H}_{1, \vartheta}^{\kappa, \omega}(\nu \mathcal{V})(\vartheta) .
\end{aligned}
$$

Proof Multiplying (35) by $\frac{1}{\omega^{\eta} \Gamma(\eta)} e^{\frac{\omega-1}{\omega}(\ln \vartheta-\ln \zeta)}(\ln \vartheta-\ln \zeta)^{\eta-1} \frac{w(\zeta)}{\zeta}, \zeta \in(1, \vartheta)$, and integrating the resultant inequality with respect to $\zeta$ over $(1, \vartheta)$, we obtain

$$
\begin{aligned}
\mathcal{H}_{1, \vartheta}^{\kappa, \omega}(\nu \mathcal{U} \mathcal{V})(\vartheta) \frac{1}{\omega^{\eta} \Gamma(\eta)} \int_{1}^{\vartheta} e^{\frac{\omega-1}{\omega}(\ln \vartheta-\ln \zeta)}(\ln \vartheta-\ln \zeta)^{\eta-1} w(\zeta) \frac{d \zeta}{\zeta} \\
\quad+\mathcal{H}_{1, \vartheta}^{\kappa, \omega}(\nu)(\vartheta) \frac{1}{\omega^{\eta} \Gamma(\eta)} \int_{1}^{\vartheta} e^{\frac{\omega-1}{\omega}(\ln \vartheta-\ln \zeta)}(\ln \vartheta-\ln \zeta)^{\eta-1} w(\zeta) \mathcal{U}(\zeta) \mathcal{V}(\zeta) \frac{d \zeta}{\zeta} \\
\geq \mathcal{H}_{1, \vartheta}^{\kappa, \omega}(\nu \mathcal{U})(\vartheta) \frac{1}{\omega^{\eta} \Gamma(\eta)} \int_{1}^{\vartheta} e^{\frac{\omega-1}{\omega}(\ln \vartheta-\ln \zeta)}(\ln \vartheta-\ln \zeta)^{\eta-1} w(\zeta) \mathcal{V}(\zeta) \frac{d \zeta}{\zeta} \\
\quad+\mathcal{H}_{1, \vartheta}^{\kappa, \omega}(\nu \mathcal{V})(\vartheta) \frac{1}{\omega^{\eta} \Gamma(\eta)} \int_{1}^{\vartheta} e^{\frac{\omega-1}{\omega}(\ln \vartheta-\ln \zeta)}(\ln \vartheta-\ln \zeta)^{\eta-1} w(\zeta) \mathcal{U}(\zeta) \frac{d \zeta}{\zeta}
\end{aligned}
$$

which by (12) gives

$$
\begin{gathered}
\mathcal{H}_{1, \vartheta}^{\kappa, \omega}(v)(\vartheta) \mathcal{H}_{1, \vartheta}^{\eta, \omega}(w \mathcal{U} \mathcal{V})(\vartheta)+\mathcal{H}_{1, \vartheta}^{\eta, \omega}(w)(\vartheta) \mathcal{H}_{1, \vartheta}^{\kappa, \omega}(v \mathcal{U} \mathcal{V})(\vartheta) \\
\geq \mathcal{H}_{1, \vartheta}^{\kappa, \omega}(v \mathcal{U})(\vartheta) \mathcal{H}_{1, \vartheta}^{\eta, \omega}(w \mathcal{V})(\vartheta)+\mathcal{H}_{1, \vartheta}^{\eta, \omega}(w \mathcal{U})(\vartheta) \mathcal{H}_{1, \vartheta}^{\kappa, \omega}(v \mathcal{V})(\vartheta)
\end{gathered}
$$

This completes the proof of Lemma 4.2.

Remark 4.2 Setting $\kappa=\eta$ in Lemma 4.2, we get Lemma 4.1.

Theorem 4.2 Let $\mathcal{U}$ and $\mathcal{V}$ be synchronous functions on $[0, \infty)$, and let $r, p, q:[0, \infty) \rightarrow$ $[0, \infty)$. Then for all $\vartheta>1, \kappa>0$, and $\omega \in(0,1]$, we have the following inequality for (12):

$$
\begin{aligned}
\mathcal{H}_{1, \vartheta}^{\kappa, \omega} & (r)(\vartheta)\left[\mathcal{H}_{1, \vartheta}^{\kappa, \omega} p(\vartheta) \mathcal{H}_{1, \vartheta}^{\eta, \omega}(q \mathcal{U} \mathcal{V})(\vartheta)+2 \mathcal{H}_{1, \vartheta}^{\kappa, \omega}(p)(\vartheta) \mathcal{H}_{1, \vartheta}^{\eta, \omega}(q \mathcal{U} \mathcal{V})(\vartheta)\right. \\
& \left.+\mathcal{H}_{1, \vartheta}^{\eta, \omega}(q)(\vartheta) \mathcal{H}_{1, \vartheta}^{\kappa, \omega}(p \mathcal{U} \mathcal{V})(\vartheta)\right] \\
& +\left[\mathcal{H}_{1, \vartheta}^{\kappa, \omega}(p)(\vartheta) \mathcal{H}_{1, \vartheta}^{\eta, \omega}(q)(\vartheta)+\mathcal{H}_{1, \vartheta}^{\eta, \omega}(p)(\vartheta) \mathcal{H}_{1, \vartheta}^{\kappa, \omega}(q)(\vartheta)\right] \mathcal{H}_{1, \vartheta}^{\kappa, \omega}(r \mathcal{U} \mathcal{V})(\vartheta) \\
\geq & \mathcal{H}_{1, \vartheta}^{\kappa, \omega}(r)(\vartheta)\left[\mathcal{H}_{1, \vartheta}^{\kappa, \omega}(p \mathcal{U})(\vartheta) \mathcal{H}_{1, \vartheta}^{\eta, \omega}(q \mathcal{V})(\vartheta)+\mathcal{H}_{1, \vartheta}^{\eta, \omega}(q \mathcal{U})(\vartheta) \mathcal{H}_{1, \vartheta}^{\kappa, \omega}(p \mathcal{V})(\vartheta)\right] \\
& +\mathcal{H}_{1, \vartheta}^{\kappa, \omega}(p)(\vartheta)\left[\mathcal{H}_{1, \vartheta}^{\kappa, \omega}(r \mathcal{U})(\vartheta) \mathcal{H}_{1, \vartheta}^{\eta, \omega}(q \mathcal{V})(\vartheta)+\mathcal{H}_{1, \vartheta}^{\eta, \omega}(q \mathcal{U})(\vartheta) \mathcal{H}_{1, \vartheta}^{\kappa, \omega}(r \mathcal{V})(\vartheta)\right] \\
& +\mathcal{H}_{1, \vartheta}^{\kappa, \omega}(q)(\vartheta)\left[\mathcal{H}_{1, \vartheta}^{\kappa, \omega}(r \mathcal{U})(\vartheta) \mathcal{H}_{1, \vartheta}^{\eta, \omega}(p \mathcal{V})(\vartheta)+\mathcal{H}_{1, \vartheta}^{\eta, \omega}(p \mathcal{U})(\vartheta) \mathcal{H}_{1, \vartheta}^{\kappa, \omega}(r \mathcal{V})(\vartheta)\right] .
\end{aligned}
$$

Proof Taking $v=p$ and $w=q$ in Lemma 4.2, we obtain

$$
\begin{aligned}
\mathcal{H}_{1, \vartheta}^{\kappa, \omega}(p)(\vartheta) \mathcal{H}_{1, \vartheta}^{\eta, \omega}(q \mathcal{U} \mathcal{V})(\vartheta)+\mathcal{H}_{1, \vartheta}^{\eta, \omega}(q)(\vartheta) \mathcal{H}_{1, \vartheta}^{\kappa, \omega}(p \mathcal{U} \mathcal{V})(\vartheta) \\
\geq \mathcal{H}_{1, \vartheta}^{\kappa, \omega}(p \mathcal{U})(\vartheta) \mathcal{H}_{1, \vartheta}^{\eta, \omega}(q \mathcal{V})(\vartheta)+\mathcal{H}_{1, \vartheta}^{\eta, \omega}(q \mathcal{U})(\vartheta) \mathcal{H}_{1, \vartheta}^{\kappa, \omega}(p \mathcal{V})(\vartheta)
\end{aligned}
$$


Multiplying (44) by $\mathcal{H}_{1, \vartheta}^{\kappa, \omega}(r)(\vartheta)$, we get

$$
\begin{aligned}
& \mathcal{H}_{1, \vartheta}^{\kappa, \omega}(r)(\vartheta)\left[\mathcal{H}_{1, \vartheta}^{\kappa, \omega}(p)(\vartheta) \mathcal{H}_{1, \vartheta}^{\eta, \omega}(q \mathcal{U} \mathcal{V})(\vartheta)+\mathcal{H}_{1, \vartheta}^{\eta, \omega}(q)(\vartheta) \mathcal{H}_{1, \vartheta}^{\kappa, \omega}(p \mathcal{U} \mathcal{V})(\vartheta)\right] \\
& \geq \mathcal{H}_{1, \vartheta}^{\kappa, \omega}(r)(\vartheta)\left[\mathcal{H}_{1, \vartheta}^{\kappa, \omega}(p \mathcal{U})(\vartheta) \mathcal{H}_{1, \vartheta}^{\eta, \omega}(q \mathcal{V})(\vartheta)+\mathcal{H}_{1, \vartheta}^{\eta, \omega}(q \mathcal{U})(\vartheta) \mathcal{H}_{1, \vartheta}^{\kappa, \omega}(p \mathcal{V})(\vartheta)\right] .
\end{aligned}
$$

Setting $v=r$ and $w=q$ in Lemma 4.2, we obtain

$$
\begin{aligned}
& \mathcal{H}_{1, \vartheta}^{\kappa, \omega}(r)(\vartheta) \mathcal{H}_{1, \vartheta}^{\eta, \omega}(q \mathcal{U} \mathcal{V})(\vartheta)+\mathcal{H}_{1, \vartheta}^{\eta, \omega}(q)(\vartheta) \mathcal{H}_{1, \vartheta}^{\kappa, \omega}(r \mathcal{U} \mathcal{V})(\vartheta) \\
& \geq \mathcal{H}_{1, \vartheta}^{\kappa, \omega}(r \mathcal{U})(\vartheta) \mathcal{H}_{1, \vartheta}^{\eta, \omega}(q \mathcal{V})(\vartheta)+\mathcal{H}_{1, \vartheta}^{\eta, \omega}(q \mathcal{U})(\vartheta) \mathcal{H}_{1, \vartheta}^{\kappa, \omega}(r \mathcal{V})(\vartheta)
\end{aligned}
$$

Multiplying (46) by $\mathcal{H}_{1, \vartheta}^{\kappa, \omega}(p)(\vartheta)$, we get

$$
\begin{aligned}
\mathcal{H}_{1, \vartheta}^{\kappa, \omega}(p)(\vartheta)\left[\mathcal{H}_{1, \vartheta}^{\kappa, \omega}(r)(\vartheta) \mathcal{H}_{1, \vartheta}^{\eta, \omega}(q \mathcal{U} \mathcal{V})(\vartheta)+\mathcal{H}_{1, \vartheta}^{\eta, \omega}(q)(\vartheta) \mathcal{H}_{1, \vartheta}^{\kappa, \omega}(r \mathcal{U} \mathcal{V})(\vartheta)\right] \\
\geq \mathcal{H}_{1, \vartheta}^{\kappa, \omega}(p)(\vartheta)\left[\mathcal{H}_{1, \vartheta}^{\kappa, \omega}(r \mathcal{U})(\vartheta) \mathcal{H}_{1, \vartheta}^{\eta, \omega}(q \mathcal{V})(\vartheta)+\mathcal{H}_{1, \vartheta}^{\eta, \omega}(q \mathcal{U})(\vartheta) \mathcal{H}_{1, \vartheta}^{\kappa, \omega}(r \mathcal{V})(\vartheta)\right] .
\end{aligned}
$$

Similarly, setting $v=r$ and $w=p$ in Lemma 4.2 and multiplying both sides of the resultant inequality by $\mathcal{H}_{1, \vartheta}^{\kappa, \omega}(q)(\vartheta)$, we obtain

$$
\begin{aligned}
\mathcal{H}_{1, \vartheta}^{\kappa, \omega}(q)(\vartheta)\left[\mathcal{H}_{1, \vartheta}^{\kappa, \omega}(r)(\vartheta) \mathcal{H}_{1, \vartheta}^{\eta, \omega}(p \mathcal{U} \mathcal{V})(\vartheta)+\mathcal{H}_{1, \vartheta}^{\eta, \omega}(p)(\vartheta) \mathcal{H}_{1, \vartheta}^{\kappa, \omega}(r \mathcal{U} \mathcal{V})(\vartheta)\right] \\
\geq \mathcal{H}_{1, \vartheta}^{\kappa, \omega}(q)(\vartheta)\left[\mathcal{H}_{1, \vartheta}^{\kappa, \omega}(r \mathcal{U})(\vartheta) \mathcal{H}_{1, \vartheta}^{\eta, \omega}(p \mathcal{V})(\vartheta)+\mathcal{H}_{1, \vartheta}^{\eta, \omega}(p \mathcal{U})(\vartheta) \mathcal{H}_{1, \vartheta}^{\kappa, \omega}(r \mathcal{V})(\vartheta)\right] .
\end{aligned}
$$

Hence we obtain the desired statement (43) by adding inequalities (45), (47), and (48).

Remark 4.3 Setting $\kappa=\eta$ in Theorem 4.2, we get Theorem 4.1.

Remark 4.4 Setting $\omega=1$ in Theorem 3.2, we get Theorem 3.4 proved by Chinchane and Pachpatte [13]

Remark 4.5 Inequalities (36) and (43) will be reversed in the following cases:

(i) The functions $\mathcal{U}$ and $\mathcal{V}$ are asynchronous on $[0, \infty)$.

(ii) The functions $r, p$, and $q$ are negative on $[0, \infty)$.

(iii) Two of the functions $r, p$, and $q$ are positive, and the third one is negative on $[0, \infty)$.

\section{Concluding remarks}

In the last few decades, fractional calculus has been extensively studied due to its wide applications in diverse fields cited in the literature. Based on that notion, the idea of generalized proportional fractional integral operators concerning the exponential function in their kernels was recently introduced by Jarad et al. [19]. Later on, the Hadamard proportional fractional integrals were introduced by Rahman et al. [32], who established certain inequalities for convex functions by employing the Hadamard proportional fractional integrals. Recently, many researchers established integral inequalities by employing generalized proportional fractional integral operators cited in the literature. In this paper, we established integral inequalities for the extended Chebyshev functional by utilizing the generalized proportional fractional and Hadamard proportional fractional integrals. Particular cases of our results can be found in the works of Dahmani [14] and Chinchane and Pachpatte [13]. 


\section{Acknowledgements}

The third author thanks Prince Sultan University for funding this research through the group "Nonlinear Analysis Methods in Applied Mathematics" (NAMAM, group number RG-DES-2017-01-17).

\section{Funding}

Not applicable.

\section{Availability of data and materials}

Not applicable.

\section{Competing interests}

The authors declare that they have no competing interests.

Authors' contributions

All authors contributed equally to the manuscript and approved the final version.

\section{Author details}

'Department of Mathematics and Statistics, Hazara University, Mansehra, Pakistan. ${ }^{2}$ Department of Mathematics, College of Arts and Sciences, Prince Sattam bin Abdulaziz University, Wadi Aldawser, 11991, Saudi Arabia. ${ }^{3}$ Department of Mathematics and General Sciences, Prince Sultan University, Riyadh, Kingdom of Saudi Arabia. ${ }^{4}$ Department of Medical Research, China Medical University, Taichung 40402, Taiwan. ${ }^{5}$ Department of Computer Science and Information Engineering, Asia University, Taichung 40402, Taiwan.

\section{Publisher's Note}

Springer Nature remains neutral with regard to jurisdictional claims in published maps and institutional affiliations.

Received: 13 March 2020 Accepted: 6 April 2021 Published online: 13 April 2021

\section{References}

1. Abdeljawad, T.: On conformable fractional calculus. J. Comput. Appl. Math. 279, 57-66 (2015). https://doi.org/10.1016/j.cam.2014.10.016

2. Abdeljawad, T., Baleanu, D.: Monotonicity results for fractional difference operators with discrete exponential kernels. Adv. Differ. Equ. 2017, 78 (2017). https://doi.org/10.1186/s13662-017-1126-1

3. Abdeljawad, T., Baleanu, D.: On fractional derivatives with exponential kernel and their discrete versions. Rep. Math. Phys. 80, 11-27 (2017). https://doi.org/10.1016/S0034-4877(17)30059-9

4. Aldhaifallah, M., Tomar, M., Nisar, K.S., Purohit, S.D.: Some new inequalities for ( $k$, s)-fractional integrals. J. Nonlinear Sci. Appl. 9(9), 5374-5381 (2016)

5. Anber, A., Dahmani, Z., Bendoukha, B.: New integral inequalities of Feng Qi type via Riemann-Liouville fractional integration. Facta Univ., Ser. Math. Inform. 27(2), 13-22 (2012)

6. Anderson, D.R., Ulness, D.J.: Newly defined conformable derivatives. Adv. Dyn. Syst. Appl. 10(2), 109-137 (2015)

7. Atangana, A., Baleanu, D.: New fractional derivatives with nonlocal and non-singular kernel: theory and application to heat transfer model. Therm. Sci. 20, 763-769 (2016). https://doi.org/10.2298/TSCI160111018A

8. Belarbi, S., Dahmani, Z:: On some new fractional integral inequalities. J. Inequal. Pure Appl. Math. 10(3), 1-12 (2009)

9. Biernacki, M.: Sur une inégalité entre les intégrales due Tchebysheff. Ann. Univ. Mariae Curie-Sklodowska 1(5), 23-29 (1951)

10. Burkill, H., Mirsky, L.: Comments on Chebysheff's inequality. Period. Math. Hung. 6, 3-16 (1975)

11. Caputo, M., Fabrizio, M.: A new definition of fractional derivative without singular kernel. Prog. Fract. Differ. Appl. 1(2), 73-85 (2015)

12. Chebyshev, P.L.: Sur les expressions approximatives des integrales definies par les autres prises entre les mêmes limite. Proc. Math. Soc. Charkov. 2, 93-98 (1882)

13. Chinchane, V.L., Pachpatte, D.B.: On some integral inequalities using Hadamard fractional integral. Malaya J. Mat. 1(1), 62-66 (2012)

14. Dahmani, Z.: New inequalities in fractional integrals. Int. J. Nonlinear Sci. 9, 493-497 (2010)

15. Dahmani, Z., Tabharit, L.: On weighted Gruss type inequalities via fractional integration. J. Adv. Res. Pure Math. 2 $31-38$ (2010)

16. Gavrea, l.: On Chebyshev type inequalities involving functions whose derivatives belong to $L_{p}$ spaces via isotonic functional. J. Inequal. Pure Appl. Math. 7(4), 121-128 (2006)

17. Houas, M.: Certain weighted integral inequalities involving the fractional hypergeometric operators. Scientia, Ser. A, Math. Sci. 27, 87-97 (2016)

18. Houas, M.: On some generalized integral inequalities for Hadamard fractional integrals. Mediterr. J. Model. Simul. 9, 43-52 (2018)

19. Jarad, F., Abdeljawad, T., Alzabut, J.: Generalized fractional derivatives generated by a class of local proportional derivatives. Eur. Phys. J. Spec. Top. 226, 3457-3471 (2017). https://doi.org/10.1140/epjst/e2018-00021-7

20. Jarad, F., Ugurlu, E., Abdeljawad, T., Baleanu, D.: On a new class of fractional operators. Adv. Differ. Equ. 2017, 247 (2017)

21. Khalil, R., Al Horani, M., Yousef, A., Sababheh, M.: A new definition of fractional derivative. J. Comput. Appl. Math. 264, 65-70 (2014)

22. Khan, T.U., Khan, M.A.: Generalized conformable fractional integral operators. J. Comput. Appl. Math. (2018). https://doi.org/10.1016/j.cam.2018.07.018

23. Kuang, J.C.: Applied Inequalities. Shandong Sciences and Technologie Press (Chinese) (2004) 
24. Losada, J., Nieto, J..: Properties of a new fractional derivative without singular kernel. Prog. Fract. Differ. Appl. 1(2), 87-92 (2015)

25. Marinkovic, S., Rajkovic, P., Stankovic, M.: The inequalities for some types q-integrals. Comput. Math. Appl. 56, 2490-2498 (2008)

26. Mitrinovic, D.S.: Analytic Inequalities. Springer, Berlin (1970)

27. Niasr, K.S., Tassadiq, A., Rahman, G., Khan, A.: Some inequalities via fractional conformable integral operators. J. Inequal. Appl. 2019, 217 (2019). https://doi.org/10.1186/s13660-019-2170-z

28. Nisar, K.S., Rahman, G., Khan, A., Tassaddiq, A., Abouzaid, M.S.: Certain generalized fractional integral inequalities. AlMS Math. 5(2), 1588-1602 (2020). https://doi.org/10.3934/math.2020108

29. Nisar, K.S., Rahman, G., Mehrez, K.: Chebyshev type inequalities via generalized fractional conformable integrals. J. Inequal. Appl. 2019, 245 (2019). https://doi.org/10.1186/s13660-019-2197-1

30. Ostrowski, A.M.: On an integral inequality. Aequ. Math. 4, 358-373 (1970)

31. Podlubny, I.: Fractional Differential Equations. Academic Press, London (1999)

32. Rahman, G., Abdeljawad, T., Jarad, F., Khan, A., Nisar, K.S.: Certain inequalities via generalized proportional Hadamard fractional integral operators. Adv. Differ. Equ. 2019, 454 (2019). https://doi.org/10.1186/s13662-019-2381-0

33. Rahman, G., Nisar, K.S., Abdeljawad, T., Ullah, S.: Certain fractional proportional integral inequalities via convex functions. Mathematics 8, 222 (2020). https://doi.org/10.3390/math8020222

34. Rahman, G., Nisar, K.S., Ghaffar, A., Qi, F.: Some inequalities of the Grüss type for conformable k-fractional integral operators. Rev. R. Acad. Cienc. Exactas Fís. Nat., Ser. A Mat. 114, 9 (2020). https://doi.org/10.1007/s13398-019-00731-3

35. Rahman, G., Nisar, K.S., Qi, F.: Some new inequalities of the Gruss type for conformable fractional integrals. AlMS Math. 3(4), 575-583 (2018)

36. Rahman, G., Ullah, Z., Khan, A., Set, E., Nisar, K.S.: Certain Chebyshev type inequalities involving fractional conformable integral operators. Mathematics 7, 364 (2019). https://doi.org/10.3390/math7040364

37. Rahmnan, G., Abdeljawad, T., Jarad, F., Nisar, K.S.: Bounds of generalized proportional fractional integrals in general form via convex functions and their applications. Mathematics 8, 113 (2020). https://doi.org/10.3390/math8010113

38. Samko, S.G., Kilbas, A.A., Marichev, O.I.: Fractional Integrals and Derivatives, Theory and Applications. Gordon \& Breach, Yverdon (1993). Edited and with a foreword by S. M. Nikol'skï, Translated from the 1987 Russian original, Revised by the authors

39. Sarikaya, M.Z., Budak, H.: Generalized Ostrowski type inequalities for local fractional integrals. Proc. Am. Math. Soc. 145(4), 1527-1538 (2017)

40. Sarikaya, M.Z., Dahmani, Z., Kiris, M.E., Ahmad, F.: (k, s)-Riemann-Liouville fractional integral and applications. Hacet. J. Math. Stat. 45(1), 77-89 (2016)

41. Set, E., Noor, M.A., Awan, M.U., Gözpinar, A.: Generalized Hermite-Hadamard type inequalities involving fractional integral operators. J. Inequal. Appl. 2017, 169 (2017)

42. Tassaddiq, A., Rahman, G., Nisar, K.S., Samraiz, M.: Certain fractional conformable inequalities for the weighted and the extended Chebyshev functionals. Adv. Differ. Equ. 2020, 96 (2020). https://doi.org/10.1186/s13662-020-2543-0

\section{Submit your manuscript to a SpringerOpen ${ }^{0}$ journal and benefit from:}

- Convenient online submission

- Rigorous peer review

- Open access: articles freely available online

- High visibility within the field

- Retaining the copyright to your article

Submit your next manuscript at $\boldsymbol{\nabla}$ springeropen.com 